\title{
Inflation from the internal volume in type IIB/F-theory compactification
}

\author{
Ignatios Antoniadis $^{a, b} 1$ Yifan Chen $^{a}{ }^{2}$, George K. Leontaris ${ }^{c}{ }^{3}$ \\ ${ }^{a}$ Laboratoire de Physique Théorique et Hautes Énergies - LPTHE, \\ Sorbonne Université, CNRS, 4 Place Jussieu, 75005 Paris, France \\ ${ }^{b}$ Albert Einstein Center, Institute for Theoretical Physics, University of Bern, \\ Sidlerstrasse 5, CH-3012 Bern, Switzerland \\ ${ }^{c}$ Physics Department, University of Ioannina \\ 45110, Ioannina, Greece
}

\begin{abstract}
We study cosmological inflation within a recently proposed framework of perturbative moduli stabilisation in type IIB/F theory compactifications on Calabi-Yau threefolds. The stabilisation mechanism utilises three stacks of magnetised 7-branes and relies on perturbative corrections to the Kähler potential that grow logarithmically in the transverse sizes of co-dimension two due to local tadpoles of closed string states in the bulk. The inflaton is the Kähler modulus associated with the internal compactification volume that starts rolling down the scalar potential from an initial condition around its maximum. Although the parameter space allows moduli stabilisation in de Sitter space, the resulting number of e-foldings is too low. An extra uplifting source of the vacuum energy is then required to achieve phenomenologically viable inflation and a positive (although tiny) vacuum energy at the minimum. We discuss a class of uplifting potentials arising from strongly coupled matter fields. In a particular case, they reproduce the effect of the new Fayet-Iliopoulos term recently discussed in a supergravity context, that can be written for a non R-symmetry $U(1)$ and is gauge invariant at the Lagrangian level.
\end{abstract}

\footnotetext{
${ }^{1}$ E-mail: antoniad@lpthe.jussieu.fr

${ }^{2}$ E-mail: yifan.chen@lpthe.jussieu.fr

${ }^{3}$ E-mail: leonta@uoi.gr
} 


\section{Introduction}

One of the key challenges in string theory cosmology is to implement a successful scenario of cosmological inflation with robust predictions of the slow-roll parameters. There are several distinct ways to realise cosmological inflation in string theory [1]. In a wide class of string inflationary models the rôle of the inflaton is played by one of the numerous moduli fields emerging in Calabi-Yau compactifications. These are the real or the imaginary part of complex structure and Kähler moduli, or those associated with the relative position of D-branes. In order to realise inflation with these candidates, however, firstly it is crucial to ensure that in the effective supergravity theory limit these moduli fields obtain the required properties. Of particular importance are the issues of moduli stabilisation and the specific constraints that should be respected by the effective potential. Thus, at the minimum of the effective potential all moduli fields should acquire positive masses squared; also, in accordance with cosmological observations confirming the accelerated expansion of the universe, a positive but tiny cosmological constant would be the optimal solution.

In the context of Calabi-Yau compactifications with background fluxes, a considerable amount of work has been devoted to constructing de Sitter (dS) vacua and stabilising the moduli fields [2, 3]. The solution of these major issues 4 are prerequisites for a successful model of string inflation. In a wide class of models in the context of type II-B string theory, the inflaton field is one of the Kähler moduli which appear in the tree-level no-scale type Kähler potential through the compactification volume [6, 7]. Their masses remain undetermined because they are absent at the tree-level superpotential. It has been suggested that they can be stabilised through non-perturbative superpotential contributions generated by gaugino condensation in some gauge group factor(s). In addition, if leading order $\alpha^{\prime}$ corrections are included in the Kähler potential they break the no-scale form and stabilise the volume. Additional contributions form D-terms or $\overline{D 3}$ branes are usually necessary to uplift the vacuum energy and generate a small positive cosmological constant.

In a previous paper [8], working in the framework of type IIB/F-theory we have investigated the possibility of obtaining dS minima and stabilising the Kähler moduli, invoking only perturbative contributions to the Kähler potential. More precisely, we have shown that these two merits are naturally feasible by relying on configurations of at least three magnetised spacetime filling 7-branes intersecting each other. We have argued that the Kähler potential receives loop corrections which display in general a logarithmic dependence on the volume of dimension two, transverse to each 7-brane [9]. Moreover, magnetised 7-branes are associated with anomalous abelian symmetries which induce world-volume dependent Fayet-Iliopoulos (FI) terms [13]. These lead to positive, moduli dependent, D-term contributions to the scalar potential which act as an uplifting mechanism giving rise to a $\mathrm{dS}$ minimum in a natural way.

In the present work, we examine the possibility to implement a successful cosmological inflationary scenario in the above framework. A decisive factor to achieve this goal is the specific structure of the effective potential of the moduli fields. It turns out that the region of parameter space leading to a dS minimum is too narrow and does not allow to obtain a successful slow-roll cosmological inflation because the number of e-foldings is too low. Thus, it is of crucial importance towards a successful prediction of the inflationary observables to implement the mechanism of perturbative moduli stabilisation with an extra uplift of the vacuum energy. Here, we ex-

\footnotetext{
${ }^{4}$ There is considerable ongoing debade and conjectures on the existence of dS vacua in string theory. For an incomplete list see for example [4. For related cosmological implications and other remarks on the conjectures see also [5].
} 
plore the consequences of a new class of FI terms in global and local supersymmetry that were proposed recently [14, 15, 16]. Their novelty is that they are gauge invariant at the Lagrangian level and, as such, can be written for any $U(1)$ that does not have to be an R-symmetry as in standard supergravity; they are however singular (non-local) in the supersymmetric limit. Also, they may involve arbitrary functions of chiral matter superfields; in the simlplest case of no new functions, there are two possibilities: one that gives a contribution to the scalar potential as the one of $\overline{D 3}$ branes but violates invariance under Kähler transformations [14, 15] and another that preserves it and amounts to a constant FI term as the one of global supersymmetry [16]. Here, as an example, we consider the second path.

The paper is organised as follows. In Section 2, we review the salient features of the geometric stabilisation mechanism in the presence of fluxes and intersecting D7-branes. We describe the effective scalar potential which comprises both F- and D-term contributions; in particular, in section 2.2, we define a canonically normalised basis for the Kähler moduli, we identify the volume with the inflaton field, and compute the mass spectrum around the minimum of the scalar potential; in subsection 2.3, we determine the parameter space for a dS minimum and study the prospects of inflation, starting with initial conditions around the maximum. We find that although the slow-roll conditions can be satisfied, the number of e-foldings are too low in the parameter region where the minimum of the potential has positive energy. An extra uplifting term is then required. In Section 3, we discuss the new FI terms that were proposed recently in a supergravity context and we choose to take into account the simplest one that preserves Kähler invariance and amounts to adding a constant uplifting in the scalar potential. Alternatively, we consider the uplifting potential arising from a nilpotent superfield describing the low energy dynamics of a strongly coupled sector. Subsequently, we study the consequences in inflation using a toy model with one Kähler modulus. In Section 4, we study the full model with three Kähler moduli and investigate the regions of the parameter space where the scalar potential is suitable for slow-roll inflation. We derive the scales associated with the inflationary period and show that our results are in agreement with the Planck 2018 measurements. Finally, Section 5 contains a summary and the conclusions of our analysis.

\section{Review of the geometric stabilisation mechanism}

In a previous paper [8], working in a type IIB/F-theory framework, we have considered a geometric configuration of three intersecting 7-branes with magnetic fluxes in order to solve the problem of Kähler moduli stabilisation in a controllable perturbative way. Fluxed 7 branes are associated with anomalous $U(1)$ symmetries which induce non-vanishing Fayet-Iliopoulos D-terms and play an instrumental rôle in achieving stabilisation of the volume and Kähler moduli. Since D-terms induce positive contributions to the scalar potential, they are essential in generating a de-Sitter minimum. Moreover, under certain conditions, to be described below, the combined effects of the D-terms and the induced loop-corrections can stabilise all Kähler moduli.

\section{$2.1 \quad$ F- and D-term potential}

In the presence of D7-branes, the Kähler potential receives quantum corrections which display a logarithmic dependence on the Kähler moduli associated with the size of their transverse dimensions [8]. This kind of quantum corrections is a generic phenomenon in string compactifications with branes/localised sources of co-dimenion two and is due to local tadpoles generated by closed strings propagating effectively in two dimensions [9]. In type-I string theory for example, loga- 
rithmic quantum corrections have been associated with the volume transverse to D7-branes [9]. This effect can be generalised to anisotropic compactifications of the six-dimensional compact space containing localised fields of co-dimension two. Based on this fact and using appropriate $T$-dualities [8] the corresponding corrections in the type IIB/F-theory framework emerge naturally in the Einstein kinetic term and consequently in the Kähler potential defined in the Einstein frame. For the simplest case of one D7 brane, let $u$ be the Kähler modulus related to the transverse volume and $\delta \equiv \gamma \ln u$ the anticipated corrections with $\gamma$ a model dependent parameter. Then, the Kähler potential is

$$
\mathcal{K}=-\ln (S-\bar{S})-2 \ln (\hat{\mathcal{V}}+\xi+\hat{\delta}),
$$

where $S$ represents the axion-dilaton modulus and $S-\bar{S}=2 i e^{-\phi}$, with $\phi$ being the 10dimensional dilaton. Furthermore,

$$
\hat{\mathcal{V}}=e^{-3 \phi / 2} \mathcal{V}, \xi=-\frac{\zeta(3)}{4(2 \pi)^{3} g_{s}^{3 / 2}} \chi \quad ; \quad \hat{\delta}=\delta g_{s}^{1 / 2}
$$

In the above formulae, $\mathcal{V}$ stands for the volume, $g_{s}=e^{\phi}$ is the string coupling, and the parameter $\xi$ stands for the $\alpha^{\prime 3}$ curvature perturbative corrections being proportional to the Euler characteristic $\chi$ of the six dimensional compact manifold [17].

It has been shown [8] however, that the stabilisation of the Kähler moduli requires at least three magnetised 7 branes intersecting each other. Thus, we consider three Kähler moduli $T_{1}, T_{2}, T_{3}$ and define the volume in terms of their real parts $\tau_{k}=\frac{1}{2}\left(T_{k}+\bar{T}_{k}\right)$,

$$
\mathcal{V}=\left(\tau_{1} \tau_{2} \tau_{3}\right)^{\frac{1}{2}}
$$

Then, the Kähler potential is

$$
\mathcal{K}=-2 \ln \left(\left(\tau_{1} \tau_{2} \tau_{3}\right)^{\frac{1}{2}}+\xi+\sum_{k=1}^{3} \gamma_{k} \ln \left(\tau_{k}\right)\right),
$$

where $\gamma_{k}$ are model dependent coefficients of order one.

The cosmological implications of the model and in particular the inflationary properties, to be discussed in the next sections, presuppose the determination of the dynamics of the moduli and other scalar fields. A necessary, although not sufficient, condition that should be fulfilled at the vacuum, is the positivity of masses-squared for all moduli fields, with the lighter being the inflaton that we identify with the total six-dimensional internal volume. Since complex structure moduli $z_{i}$ are assumed to be present in the superpotential, they can be stabilised by the conditions $D_{z_{i}} \mathcal{W}=0$, thus we only need to deal here with the three Kähler moduli $\tau_{k}$. In [8] the minimisation conditions were considered with respect to the total volume $\mathcal{V}$ and two ratios of the three Kähler moduli $\tau_{k}$.

We first recall the details for the minimisation with respect to the total volume of the corresponding F-term scalar potential $V_{F}$. Assuming for simplicity $\gamma_{1}=\gamma_{2}=\gamma_{3} \equiv \gamma_{\tau}$, we can write the Kähler potential (3) as follows

$$
\mathcal{K}=-2 \log \left(\mathcal{V}+\xi+2 \gamma_{\tau} \ln (\mathcal{V})\right)
$$

The $\alpha^{\prime}$ corrections, $\xi$, can be absorbed into the logarithmic term by replacing the implicit string scale unit inside $\ln \mathcal{V}$ by a new parameter $\mu$

$$
\xi+2 \gamma_{\tau} \ln (\mathcal{V})=2 \gamma_{\tau} \ln (\mu \mathcal{V}), \quad \mu=e^{\frac{\xi}{2 \gamma_{\tau}}}
$$


Computing the corresponding F-term potential, we find

$$
V_{F}=-3 \gamma_{\tau} W_{0}^{2} \frac{2\left(\gamma_{\tau}+2 \mathcal{V}\right)+\left(4 \gamma_{\tau}-\mathcal{V}\right) \ln (\mu \mathcal{V})}{\left(\mathcal{V}+2 \gamma_{\tau} \ln (\mu \mathcal{V})\right)^{2}\left(6 \gamma_{\tau}^{2}+\mathcal{V}^{2}+8 \gamma_{\tau} \mathcal{V}+\gamma_{\tau}\left(4 \gamma_{\tau}-\mathcal{V}\right) \ln (\mu \mathcal{V})\right)} .
$$

This rather complicated formula obscures the properties of the vacuum of the potential, however, in the large volume limit and relatively small logarithmic corrections, this can be approximated by

$$
V_{F} \approx 3 W_{0}^{2} \gamma_{\tau} \frac{\ln (\mu \mathcal{V})-4}{\mathcal{V}^{3}}
$$

It can be readily inferred that a minimum of the potential (7) exists as long as $\gamma_{\tau}<0$. Then, imposing the condition $\frac{d V_{F}}{d \mathcal{V}}=0$ we find that the minimum is localised at $\mathcal{V}_{\min }=e^{\frac{13}{3}} / \mu$. Substitution of $\mathcal{V}_{\text {min }}$ into the potential gives an AdS minimum

$$
\left(V_{F}\right)_{\min }=\frac{\gamma_{\tau} W_{0}^{2}}{\mathcal{V}^{3}}<0
$$

since $\gamma_{\tau}<0$, as we have already assumed. Furthermore, the flatness conditions become

$$
D_{\mathcal{V}} W_{0}=-2 \frac{W_{0}^{2}}{\mathcal{V}_{\min }} \neq 0
$$

and therefore supersymmetry is spontaneously broken.

The incorporation of $D 7$ fluxed branes into the theory, induces also D-term contributions to the effective potential. Hence, in addition to the F-term potential given above, we take into account the following contribution

$$
V_{D}=\sum_{a=1}^{3} \frac{d_{a}}{\tau_{a}}\left(\frac{\partial \mathcal{K}}{\partial \tau_{a}}\right)^{2} \approx \sum_{a=1}^{3} \frac{d_{a}}{\tau_{a}^{3}},
$$

in the large volume limit.

Including F- and D-term contributions, in the large volume expansion, the effective potential can be approximated as follows

$$
V_{\mathrm{eff}} \approx 3 W_{0}^{2} \gamma_{\tau} \frac{\ln (\mu \mathcal{V})-4}{\mathcal{V}^{3}}+\frac{d_{i}}{\tau_{i}^{3}}+\frac{d_{j}}{\tau_{j}^{3}}+\frac{d_{k}\left(\tau_{i} \tau_{j}\right)^{3}}{\mathcal{V}^{6}}, \quad i \neq j \neq k \neq i
$$

The remaining two minimisation conditions determine the ratios between the moduli, $\left(\frac{\tau_{i}}{\tau_{k}}\right)^{3}=$ $\frac{d_{i}}{d_{k}}$. Expressed in terms of the stabilised total volume $\mathcal{V}$, these can be written as

$$
\tau_{i}^{3}=\left(\frac{d_{i}^{2}}{d_{k} d_{j}}\right)^{\frac{1}{3}} \mathcal{V}^{2} .
$$

Inserting the above in $V_{\text {eff }}$ we obtain the simple form

$$
V_{\mathrm{eff}} \approx \gamma \frac{\ln (\mu \mathcal{V})-4}{\mathcal{V}^{3}}+\frac{d}{\mathcal{V}^{2}}
$$

where for simplicity we have introduced the new constants

$$
\begin{aligned}
d & =3\left(d_{1} d_{2} d_{3}\right)^{\frac{1}{3}}, \\
\gamma & =3 W_{0}^{2} \gamma_{\tau} .
\end{aligned}
$$


It was shown in 8 ] that the above scalar potential possesses a dS minimum in some region of the parameter space. However since most of the computations were done numerically and the explicit mass spectrum which is necessary for the present study in cosmology was not computed, we perform these computations in the next two subsections below and work out the consequences for inflation.

\subsection{Normalised fields and mass eigenstates}

Cosmological inflation is conveniently studied by performing a suitable transformation of the inflaton field in order to obtain a canonically normalised kinetic term. Obviously, this is also needed for the computation of the mass spectrum.

We start by first noting that in the limit where the volume $\mathcal{V}$ is much larger than the loop correction term $\gamma_{i} \ln \left(\frac{\mathcal{V}}{\tau_{i}}\right)$, we can use the no-scale Kähler potential $\mathcal{K}_{N S}$

$$
\mathcal{K}_{N S}=-2 \ln (\mathcal{V})=-\sum_{i} \ln \left(T_{i}+\bar{T}_{i}\right)=-\sum_{i} \ln \left(\tau_{i}\right),
$$

to derive the kinetic terms. We obtain

$$
\mathcal{K}_{T_{I} \bar{T}_{J}} \partial T_{I} \partial \bar{T}_{J}=\sum_{i} \frac{\partial T_{i} \partial \bar{T}_{i}}{\left(T_{i}+\bar{T}_{i}\right)^{2}}=\sum_{i} \frac{\left(\partial \tau_{i}\right)^{2}}{4 \tau_{i}^{2}}+\cdots=\sum_{i} \frac{1}{2}\left(\partial t_{i}\right)^{2}+\cdots,
$$

where $\{\cdots\}$ correspond to the kinetic terms of axions absorbed by the $U(1)$ fields related to the magnetised branes with the corresponding D-terms, and

$$
t_{i}=\frac{1}{\sqrt{2}} \ln \left(\tau_{i}\right)
$$

are the normalised real scalar fields. Since we have three Kähler moduli, it would be better to switch to the mass eigenstates and find the lightest one. One option is taking the total volume (2) and two other perpendicular directions:

$$
\begin{aligned}
t & =\frac{1}{\sqrt{3}}\left(t_{1}+t_{2}+t_{3}\right)=\frac{\sqrt{6}}{3} \ln (\mathcal{V}), \\
u & =\frac{1}{\sqrt{2}}\left(t_{1}-t_{2}\right) \\
v & =\frac{1}{\sqrt{6}}\left(t_{1}+t_{2}-2 t_{3}\right) .
\end{aligned}
$$

The corresponding F-term and D-term components of the scalar potential in this new basis are

$$
\begin{aligned}
V_{F} & \simeq \frac{\gamma}{2} e^{-\frac{3 \sqrt{6}}{2} t}(\sqrt{6} t+2 \ln (\mu)-8) \\
V_{D} & \simeq \frac{d_{1}}{\tau_{1}^{3}}+\frac{d_{2}}{\tau_{2}^{3}}+\frac{d_{3}}{\tau_{3}^{3}} \\
& =d_{1} e^{-\sqrt{6} t-\sqrt{3} v-3 u}+d_{2} e^{-\sqrt{6} t-\sqrt{3} v+3 u}+d_{3} e^{-\sqrt{6} t+2 \sqrt{3} v}
\end{aligned}
$$

where the $\simeq \operatorname{sign}$ is used for the volume suppressed corrections.

A deviation from the assumed universal value $\gamma_{i}=\gamma_{\tau}$ contributes an additional term linear in $t_{i}$ inside the bracket of eq. (19). In the $t, u, v$ basis, the additional part of the potential can be written as:

$$
\delta V_{F} \simeq e^{-\frac{3 \sqrt{6}}{2} t}\left(\delta \gamma_{t} t+\delta \gamma_{u} u+\delta \gamma_{v} v\right)
$$


As long as $\delta \gamma_{u}$ and $\delta \gamma_{v}$ are smaller than the universal $\gamma$, the $u$ and $v$ dependent parts in (22) are negligible compared to the D-term potential (21), and we ignore them. Then, together with $\ln (\mu)$, eq. 19$)$ can be written as

$$
\begin{aligned}
V_{F} & =\frac{\gamma}{2} e^{-\frac{3 \sqrt{6}}{2} t}(A t+B) \\
& \sim \frac{\gamma}{2} e^{-\frac{3 \sqrt{6}}{2} t}(\sqrt{6} t-8+2 x)
\end{aligned}
$$

where $A$ and $B$ are $t$-independent constants. The non-universal parts contribute to $A$ and $B$ while $\ln (\mu)$ contributes to $B$ only. Since the overall scale is irrelevant in slow-roll calculation, we can rescale $A$ to be the same as in eq. $(19)$ and the parameter $x$ in eq. (24) contains both non-universal terms and $\ln (\mu)$. In the next subsection, we will see that the existence of a non-vanishing $x$ will only shift the potential towards larger t. Furthermore, the calculation of the slow-roll parameters are dominated by the exponential part $e^{-\frac{3 \sqrt{6}}{2} t}$. Thus, for simplicity we neglect both non-universal terms and $\ln (\mu)$ in eq. 19.

The vanishing of the first derivatives with respect to the moduli $u$ and $v$ of $(21)$ lead to the minimisation conditions

$$
\begin{aligned}
& u_{0}=\frac{1}{6} \ln \left(\frac{d_{1}}{d_{2}}\right) \\
& v_{0}=\frac{1}{6 \sqrt{3}} \ln \left(\frac{d_{1} d_{2}}{d_{3}^{2}}\right) .
\end{aligned}
$$

At these positions, the D-term potential becomes

$$
\left.V_{D}\right|_{u_{0}, v_{0}}=e^{-\sqrt{6} t} 3\left(d_{1} d_{2} d_{3}\right)^{\frac{1}{3}}=d e^{-\sqrt{6} t},
$$

where $d$ is defined in (11).

The masses of $t, u$ and $v$ can be calculated by taking the second derivatives of equations 19 ) and (21)

$$
\begin{aligned}
& m_{t}^{2}=V_{F t t}+\left.V_{D t t}\right|_{u_{0}, v_{0}}=\frac{9}{4} \gamma e^{-\frac{3 \sqrt{6}}{2} t}(3 \sqrt{6} t-28)+6 d e^{-\sqrt{6} t}, \\
& m_{u}^{2}=\left.V_{D u u}\right|_{u_{0}, v_{0}}=m_{v}^{2}=\left.V_{D v v}\right|_{u_{0}, v_{0}}=6 d e^{-\sqrt{6} t},
\end{aligned}
$$

with $\gamma$ defined in 12 .

The question now arises whether the potential meets the requirements for a slow-roll single field inflation where the inflaton field is played by some Kähler modulus. It is readily realised that the most natural candidate is the total volume. Hence, in the regime where the moduli masses satisfy the condition

$$
\left|m_{t}^{2}\right| \ll m_{u}^{2}=m_{v}^{2}
$$

we can use the effective potential

$$
V_{\text {eff }}=V_{F}+\left.V_{D}\right|_{u_{0}, v_{0}}=\frac{1}{2} \gamma e^{-\frac{3 \sqrt{6}}{2} t}(\sqrt{6} t-8)+d e^{-\sqrt{6} t},
$$

to describe the dynamics of the modulus $t$ related to the total volume via (16). 


\subsection{A first step towards inflation}

To examine the implications on inflation, firstly we proceed with an analysis of the properties of the scalar potential. At the classical level, we already know that the Kähler moduli dependent scalar potential vanishes identically. Then, the $t$-dependent potential $V_{\text {eff }}$ in eq. $(29)$ generated from quantum corrections, in the absence of any other effects -including the FI-terms to be discussed later- should vanish as $t \rightarrow \infty$. Moreover, the requirement for a dS minimum implies that its zero value at infinity should be approached from positive values $\lim _{t \rightarrow \infty} V_{\text {eff }}(t) \rightarrow 0^{+}$. In the simplest case, this can be realised when the potential has a minimum and a maximum at finite values of the volume modulus $t$.

We start the analysis with the minimisation of the rescaled potential in eq. (24)

$$
V_{\text {eff }}=\frac{1}{2} \gamma e^{-\frac{3 \sqrt{6}}{2} t}(\sqrt{6} t+2 x-8)+d e^{-\sqrt{6} t}
$$

with respect to $t$. In searching for extrema of the potential, we put its first derivative equal to zero and obtain the equation

$$
V_{\mathrm{eff}}^{\prime}=\frac{d V_{\mathrm{eff}}}{d t}=-\frac{\sqrt{6}}{4} e^{-\frac{3 \sqrt{6}}{2} t}\left(4 d e^{\frac{\sqrt{6}}{2} t}+\gamma(3 \sqrt{6} t+6 x-26)\right)=0 .
$$

This equation has two solutions which correspond to a minimum and a maximum of the potential. Indeed, defining

$$
w=\frac{13}{3}-x-\frac{\sqrt{6}}{2} t
$$

the above condition takes the form

$$
w e^{w}=z, \text { with } z=\frac{2}{3} \frac{d}{\gamma} e^{\frac{13}{3}-x} .
$$

For real values, $w$ should be restricted by the lower bound $w>-e^{-1}$. Moreover, the solution of $(32)$ is double-valued in the range $\left(-e^{-1}, 0\right)$, and it is expressed in terms of the Lambert $W$-function. This range in turn implies the following bounds on the ratio of the parameters $d$ and $\gamma$

$$
-\frac{3 e^{-\frac{16}{3}}}{2} \simeq-0.007242<\frac{d}{\gamma} e^{-x}<0
$$

The two branches of the Lambert $W$-function are denoted with $W_{0}(z), W_{-1}(z)$ and determine the minimum and the maximum of the canonically normalised 'volume' respectively, through the equations

$$
\begin{aligned}
t_{\min } & =\sqrt{\frac{2}{3}}\left(\frac{13}{3}-x-W_{0}\left(\frac{2 d e^{\frac{13}{3}-x}}{3 \gamma}\right)\right) \\
t_{\max } & =\sqrt{\frac{2}{3}}\left(\frac{13}{3}-x-W_{-1}\left(\frac{2 d e^{\frac{13}{3}-x}}{3 \gamma}\right)\right) .
\end{aligned}
$$

The region (34) is further restricted from above, due to the requirement for a positive energy minimum of the scalar potential. Putting eq. (35) into eq. (30), one gets

$$
V_{\min }^{\mathrm{eff}}=\frac{1}{3} e^{-\frac{3 \sqrt{6}}{2} t_{\min }}\left(\gamma+d e^{\frac{\sqrt{6}}{2}} t_{\min }\right) \geq 0
$$


which can be solved to give the numerical bound $\frac{d}{\gamma} e^{-x} \leq-0.006738$. Hence, finally the range is restricted to be

$$
-0.007242<\frac{d}{\gamma} e^{-x} \leq-0.006738
$$

as can be seen in Fig. 1

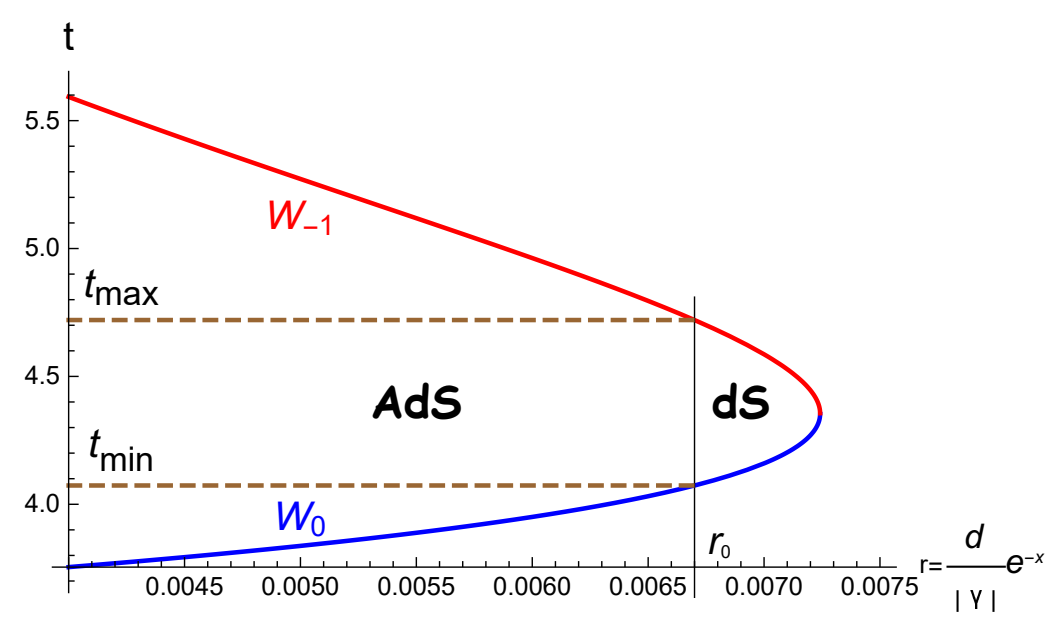

Figure 1: The canonically normalised volume plotted as a function of the parameter ratio $r=\frac{d}{|\gamma|} e^{-x}$. The two different colours of the curve correspond to the two different branches of the Lambert function(see text). For this case de Sitter minima are only possible for $r \geq r_{0}$. The positions of the minimum and maximum of the scalar potential are denoted with $t_{\min }, t_{\max }$ respectively.

Given the fact that the form of the potential (30) ensures a positive vacuum energy, in the following we would like to examine whether it is also sufficient to accommodate inflation. We will see that this is not possible and new contributions have to be taken into account, which will be discussed in the subsequent section.

Indeed, the region (38) is too restrictive to accommodate the number of e-foldings required in the slow roll inflationary scenario. To show this, it suffices to work out the limiting case $\frac{d}{|\gamma|} e^{-x}=r_{0} \approx 0.006738$, corresponding to the upper bound of 38 where the two extrema of the effective potential are maximally separated. In this case the minımum and the maximum of the potential are found to be

$$
t_{\min } \approx 4.07-\sqrt{\frac{2}{3}} x, t_{\max } \approx 4.72-\sqrt{\frac{2}{3}} x
$$

The ratio between the potential $V_{\text {eff }}(30)$ and its derivative $V_{\text {eff }}^{\prime}(31)$

$$
\frac{V_{\text {eff }}}{V_{\text {eff }}^{\prime}}=-\frac{1}{\sqrt{6}}+\frac{3\left(t+\sqrt{\frac{2}{3}} x\right)-5 \sqrt{6}}{3\left(3 \sqrt{6}\left(t+\sqrt{\frac{2}{3}} x\right)+4 r e^{\sqrt{\frac{3}{2}}\left(t+\sqrt{\frac{2}{3}} x\right)}-26\right)}
$$

is always a function of $\left(t+\sqrt{\frac{2}{3}} x\right)$. Using this formula, we find that the number of e-foldings for this limiting case are within the bound

$$
N \leq \int_{t_{\min }}^{t_{\max }} \frac{V_{\mathrm{eff}}}{V_{\mathrm{eff}}^{\prime}} d t \approx \mathcal{O}(1)
$$

which is independent of $x$. 
The number of e-foldings is an order of magnitude smaller than required. The reason is that in a large part of the parameter space $\frac{d}{\gamma} \in[-0.006738-0.0]$ where the difference $\Delta t=t_{\text {max }}-t_{\text {min }}$ would be sufficient to give the observed number of e-foldings, the potential displays an AdS minimum. Therefore, we infer that it is impossible to generate enough e-foldings without a new contribution which will uplift the potential.

\section{Additional Uplifting}

\subsection{Contributions from Fayet-Iliopoulos terms}

From the discussion in the previous section, we have realised that the existence of an uplifting term is of crucial importance to implement the inflationary scenario in the present model. Constant Fayet-Iliopoulos D-terms would be a possible tool to use in this direction. However, unlike in global supersymmetry, such terms are highly restricted in supergravity and even more in string theory. Part of the problem is due to the fact that at the Lagrangian level a constant FI-term is not gauge invariant. Moreover, a constant FI-term in supergravity can be written only for a $U(1)$ that gauges the $R$-symmetry and thus a constant term in the superpotential is not allowed. In the absence of matter this leads to the Friedman supergravity model [18 that breaks supersymmetry in de Sitter (dS) space without superpotential and, thus, without explicit gravitino mass. It is unclear how this model (or generalisations of it) could be realised in string theory.

Recently, Ref. [14 has initiated a new class of FI-terms in supergravity that avoid the above problems. Indeed, a novel FI-term was proposed which is gauge invariant at the Lagrangian level and can be written for a non-R $U(1)$. Although its form appears to be non-local in superspace, it can be expanded in components if the D-auxiliary field has non-vanishing expectation value (VEV), leading to the standard FI-term of global supersymmetry plus fermionic contributions involving the $U(1)$ gaugino. In the case where supersymmetry is broken only by this D-term, the gaugino is absorbed by the gravitino that becomes massive due to the super-Higgs mechanism and the Lagrangian is reduced to the usual FI-term. In the absence of matter, one obtains the Friedman model extended by a constant superpotential that amounts to a gravitino mass-term $m_{3 / 2}$ as independent parameter from the cosmological constant that shifts the vacuum energy above the anti-de Sitter lower bound of $-3 m_{3 / 2}^{2}$. In the presence of neutral matter, the new D-term leads to a scaler potential $\sim e^{2 \mathcal{K} / 3}$, with $\mathcal{K}$ the Kähler potential, that breaks Kähler invariance of standard $N=1$ supergravity explicitly. Interestingly, for the case of the total volume, this potential is similar to the one of $\overline{D 3}$ brane.

The new FI-term has been extended in several ways. In particular, it has been shown that it can be written in the presence of charged matter superfields and on top of the standard FI-term, even in the case of R-symmetry [15. Moreover, it is possible to consistently modify it so that Kähler invariance is preserved [16], in which case it is constant and the vacuum energy is uplifted by an arbitrary constant value. On the other hand, new functions of chiral matter field may be involved in more general extensions of this term [19, 20, 15].

It is an open interesting question wether such terms can appear in the effective supergravity of string compactifications. Here we will consider the consequences of adding a constant FI-term as an uplifting vacuum energy source in the effective supergravity that allows Kähler moduli stabilisation due to perturbative string corrections, discussed above. We will assume that this FI-term is associated with an extra $U(1)$ whose gauge coupling is fixed by 3 -form fluxes, such as in the case of an effective 3 -brane. Denoting this constant contribution with $V_{u p}$ and taking 
into account the F- and D- contributions discussed above, the final form of the scalar potential becomes:

$$
V_{\mathrm{eff}} \approx W_{0}^{2} \gamma_{\tau} \frac{\ln (\mathcal{V})-4}{\mathcal{V}^{3}}+\frac{d}{\mathcal{V}^{2}}+V_{u p}
$$

\subsection{Contribution from a nilpotent supefield}

Alternatively, one could consider the uplifting potential from strongly coupled matter fields [21, 22]. In our scenario, we expect these matter fields to be localised at the intersection points of the three 7-branes. After integrating out the heavy modes, the effective Kähler potential and superpotential contain an additional nilpotent superfield $X$ with $X^{2}=0$.

$$
\begin{aligned}
\Delta \mathcal{K} & =\frac{X \bar{X}}{\tau_{1}^{n_{1}} \tau_{2}^{n_{2}} \tau_{3}^{n_{3}}}, \\
\Delta \mathcal{W} & =f X,
\end{aligned}
$$

where $f$ is a dimension 2 constant related to the dynamical supersymmetry breaking sector, and $n_{i}$ are rational numbers correspondiing to modular weights of the dual heterotic theory. In orbifold compactifications they are given by a sum of integers (number of oscillators) and orbifold twists (see for instance [23]).

We assume the universal modular weights $n_{1}=n_{2}=n_{3}=n$ leading to the additional F-term potential in the large volume limit is

$$
\Delta V_{F}=\frac{f^{2}}{\left(\tau_{1} \tau_{2} \tau_{3}\right)^{1-n}}=\frac{f^{2}}{\mathcal{V}^{2-2 n}} .
$$

Here we consider the interesting case where $n=1-\frac{1}{2} \nu$ with $\nu$ being an orbifold twist with possible values in supersymmetric orbifolds $\nu=0$ or multiples of $\frac{1}{12}$. If we take $\nu=0$, this shares the same form as the uplifting term from the new D-term in eq. 41) with $V_{u p}=f^{2}$.

We have checked that $\nu=\frac{1}{12}$ provides a realistic model of inflation. However, below we present the analysis for the simplest case $\nu=0$ which reproduces also the contribution on the new FI term in (41). Thus, below we will use the effective potential

$$
V_{F}+\left.V_{D}\right|_{u_{0}, v_{0}}+V_{u p}=\frac{1}{2} e^{-\frac{3 \sqrt{6}}{2} t}(\sqrt{6} t-8) \gamma+e^{-\sqrt{6} t} d+V_{u p}
$$

to describe the dynamics of the modulus $t$ related to the total volume via (16), where $\gamma$ is defined in eq. (12).

\subsection{A toy model: One Kähler modulus inflation}

Before analysing the fully-fledged case, we can first consider a toy model which contains only one Kähler modulus, and turn on the logarithmic correction and the cosmological constant. The corresponding Kähler potential and the effective potential in the large volume limit reads

$$
\begin{aligned}
\mathcal{K}_{\text {toy }} & =-2 \ln \left(\tau^{\frac{3}{2}}+\gamma_{\text {toy }} \ln (\tau)\right) \\
V_{\text {toy }} & \simeq \gamma_{\text {toy }} \mathcal{W}_{0}^{2} \frac{3 \ln (\tau)-8}{2 \tau^{\frac{9}{2}}}+V_{u p} \\
& =\gamma_{\text {toy }} \mathcal{W}_{0}^{2} \frac{\sqrt{6} \phi-8}{2} e^{-\sqrt{\frac{27}{2}} \phi}+V_{\text {up }}
\end{aligned}
$$

\footnotetext{
${ }^{5}$ The nilpotency condition arises as an effective description of the models of [21, 22] after integrating out the scalar component in $X$.
} 
where we use in the last line the canonically normalised field $\phi=\sqrt{\frac{3}{2}} \ln (\tau)$. Eq. 48 gives a form similar to Starobinsky-like inflation and the slow-roll parameters fit well with the observations as shown in Fig. (2).

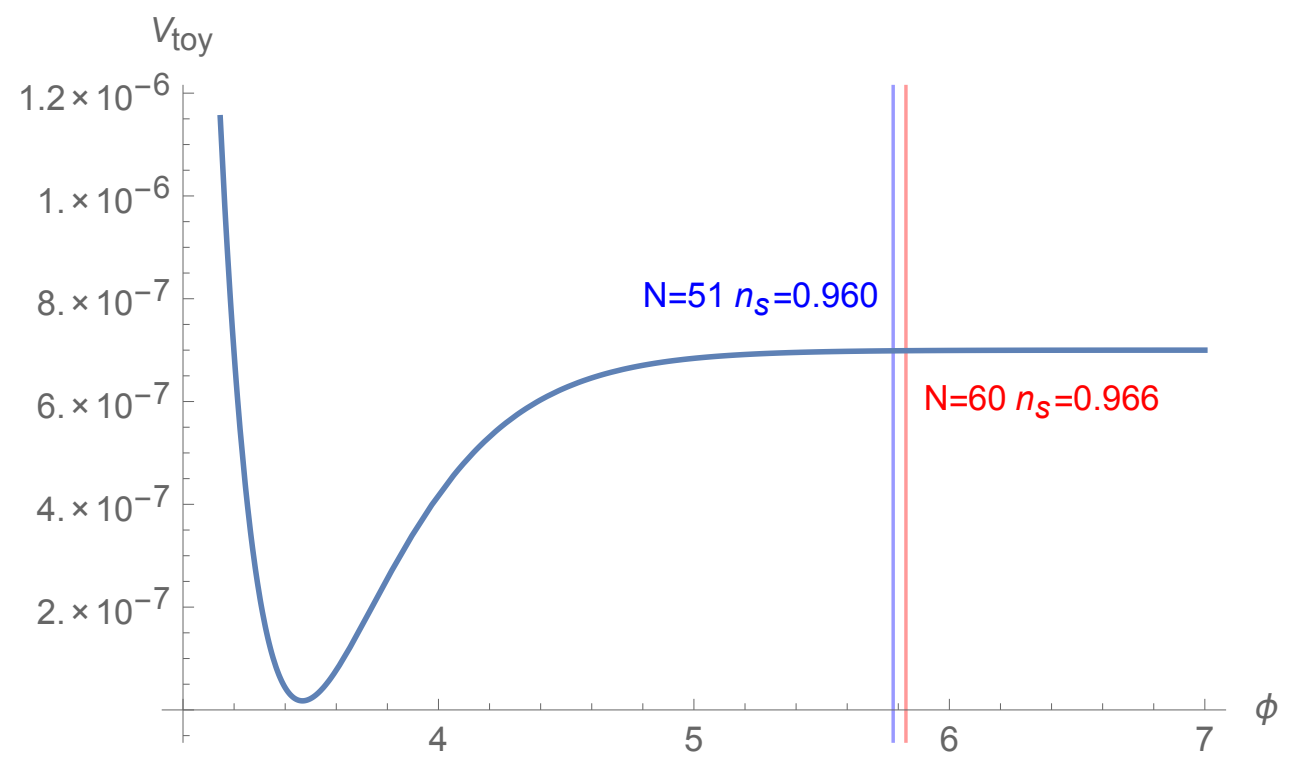

Figure 2: The effective potential of eq. 48) with $\gamma=-0.7, \mathcal{W}_{0}=1, V_{u p}=7 \times 10^{-7}$.

However, for the realistic case, as discussed in [8], one needs at least three Kähler moduli to induce the logarithmic corrections. Thus we need also to stabilise the other two moduli during inflation.

\section{Inflation from the total volume}

In the following, we analyse the general case including corrections to the effective potential due to all FI-terms.

Inflation should occur between the maximum $t_{\max }$ and the minimum, $t_{\min }$ of eqs. 35,36 ). Between them, we can define another two points. The first one is the ending point of inflation, which corresponds to the breaking of the slow-roll condition:

$$
t_{\text {end }}=\max \left\{\left.t\right|_{\frac{1}{2}\left(\frac{V^{\prime}}{V}\right)^{2} \simeq 1}, t \frac{\mid \frac{\left|V^{\prime \prime}\right|}{V} \simeq 1}{}\right\},
$$

where the derivatives $V^{\prime}, V^{\prime \prime}$ are taken with respect to $t$. The second point is the one corresponding to the anticipated number of e-foldings $N_{*} \sim 50$ to 60 , where $N_{*}$ is given by the formula

$$
N_{*}=\int_{t_{\text {end }}}^{t_{*}} \frac{V}{V^{\prime}} d t
$$

In addition, at the same point $t_{*}\left(N_{*}\right)$, the spectral index should satisfy the values from observations

$$
n_{s}=1-6 \epsilon+2 \eta=1-\left.3\left(\frac{V^{\prime}}{V}\right)^{2}\right|_{t_{*}}+\left.2 \frac{V^{\prime \prime}}{V}\right|_{t_{*}} .
$$


Obviously, the four positions should satisfy the inequality:

$$
t_{\text {min }}<t_{\text {end }}<t_{*} \leq t_{\max } .
$$

Due to the presence of the other two scalar fields, $u$ and $v$, we should consider the multi-fields effect which happens when the inflaton is no longer the lightest scalar, and the condition eq. (28) is violated:

$$
t_{m f} \in\left\{t|| m_{t}^{2} \mid \geq m_{u / v}^{2}\right\}
$$

We now study the parameter space to find regions satisfying all the aforementioned constraints. In the large volume limit, the inflationary potential (45) has three parameters. There are three conditions to fix each one of them: the first is that at the minimum, the vacuum energy is almost zero; the second is that at the point $t_{*}\left(N_{*}\right)$ corresponding to a given number $N_{*}$ of e-foldings, the spectral index $n_{s}$ should fit the observed value; the last one is the scalar power spectrum amplitude $A_{s}$. Since the three parameters are all linear in the large volume limit, one can first absorb the overall scale and fix $\gamma$ to be a small negative number. In fact, the choice of $\gamma$, although redundant, is convenient for the numerical analysis. Thus, taking $\gamma=-0.1$, the scalar potential can be written as

$$
\begin{aligned}
V_{\text {inf }} & =V_{F}+\left.V_{D}\right|_{u_{0}, v_{0}}+V_{u p} \\
& =a\left(-\frac{1}{20} e^{-\frac{3 \sqrt{6}}{2} t}(\sqrt{6} t-8)+e^{-\sqrt{6} t} d+V_{u p}\right),
\end{aligned}
$$

in which $a$ is the overall constant which can be fixed by the amplitude later. The almost zero vacuum energy in the minimum can fix another parameter. We choose $V_{u p}$ to be the one fixed and write it as a function of $d, V_{u p}=V_{u p}(d)$. Finally, the spectral index $n_{s}$ observed fixes the last parameter, in terms of the e-fold number $N_{*}: d\left(n_{s}, N_{*}\right)$. Numerically we solve the condition eq. (51) and find that $d\left(N_{*}\right)$ is a monotonically increasing function in the range $50 \leq N_{*} \leq 60$ for $n_{s}=0.9605$ which is within the $1 \sigma$ region of Planck 2018 TT,TE,EE+lowE measurement [24. The results are shown in Table 1 .

\begin{tabular}{|c|c|c|c|c|c|c|c|c|c|c|}
\hline$N_{*}$ & $\gamma$ & $\mathrm{d}$ & $V_{u p}$ & $a$ & $r$ & $t_{\min }$ & $t_{\text {end }}$ & $t_{*}$ & $t_{\max }$ & $t_{m f}$ \\
\hline 53 & -0.1 & 0.00015 & $5.85 \times 10^{-8}$ & 0.000111 & 0.00021 & 3.57 & 4.58 & 5.82 & 6.76 & $3.91 \leq t \leq 6.33$ \\
\hline 60 & -0.1 & 0.00026 & $4.20 \times 10^{-8}$ & 0.000074 & 0.00010 & 3.63 & 4.63 & 5.79 & 6.14 & $4.05 \leq t \leq 5.61$ \\
\hline
\end{tabular}

Table 1: The predictions for two choices of the parameters $\gamma, d, V_{u p}, \alpha$ involved in the scalar potential (54), for fixed value of the spectral index $n_{s}=0.9605$.

We show one example for $N_{*}=60$ in Fig. 3 . 


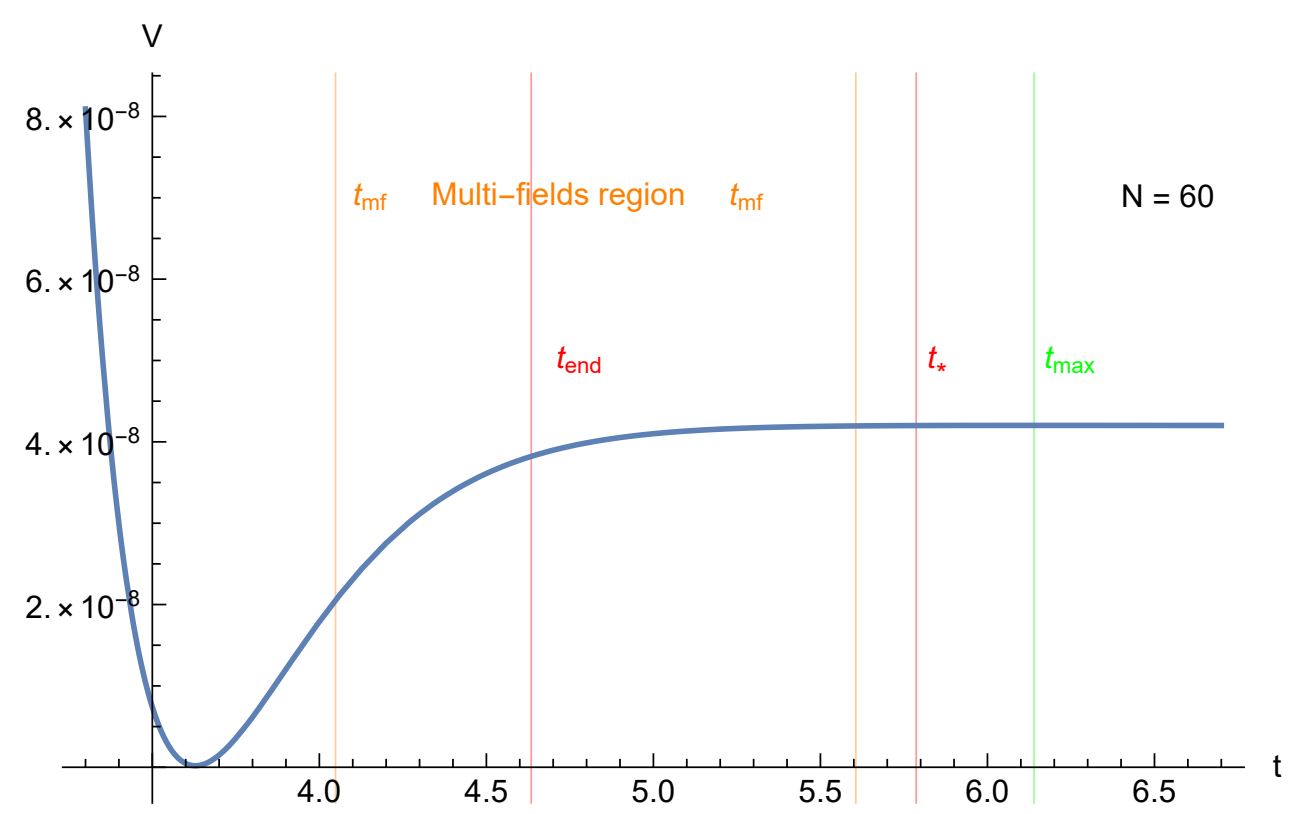

Figure 3: The potential 45 with the parameters from Table 1 for $n_{s}=0.9605, N_{*}=60$

Between the two orange lines is the area that the inflaton $t$ is not the lightest Kähler modulus. This can be seen from the ratio of the different mass scales in Fig. 4 .

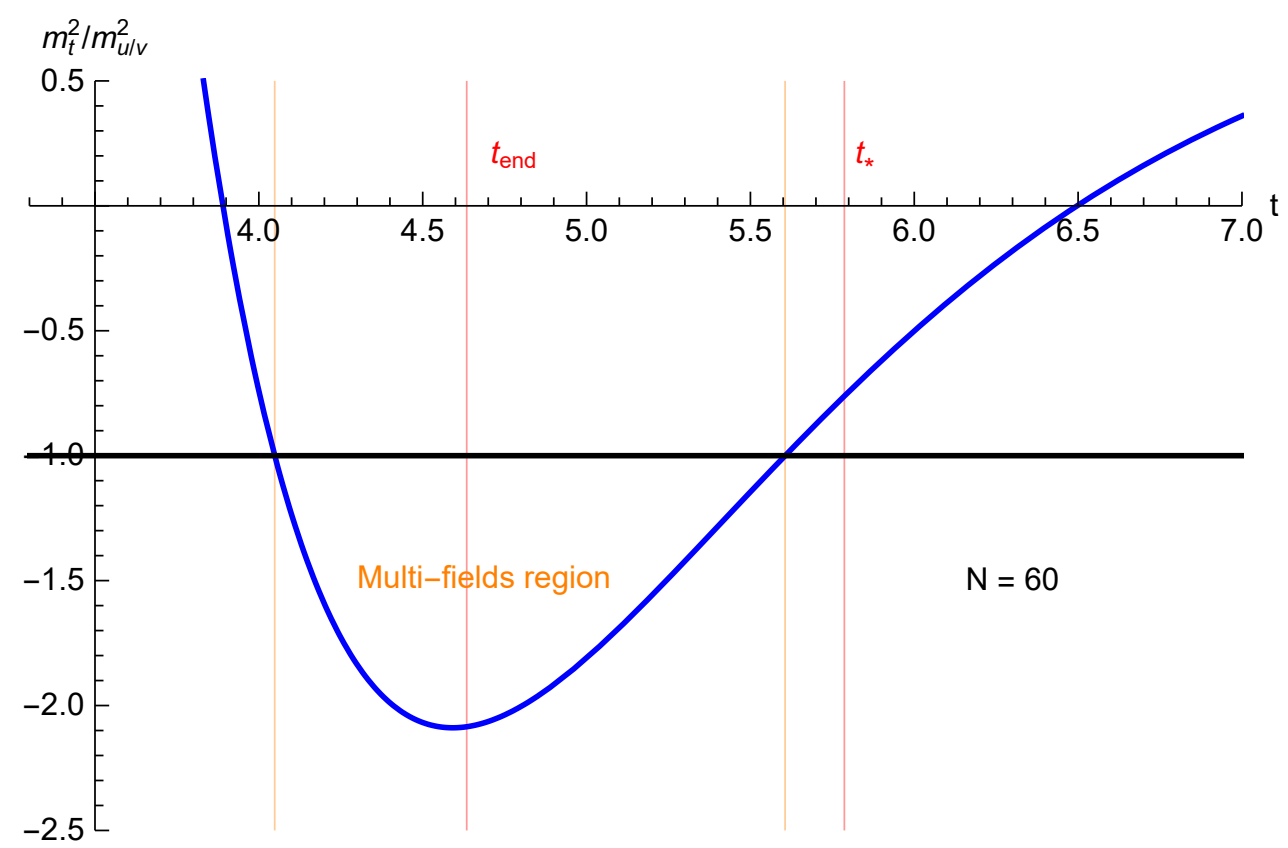

Figure 4: The mass ratio between the inflaton $t$ and the other two Kähler moduli for $n_{s}=0.9605, N_{*}=60$. The multi-field region is among the two orange lines.

As we decrease $N_{*}$ by lowering the parameter $d$, we can see that the multi-field region becomes larger. In the example of $N_{*}=53$ in Table 1, the multi-field region covers the whole inflation period as shown in Fig. 5. 


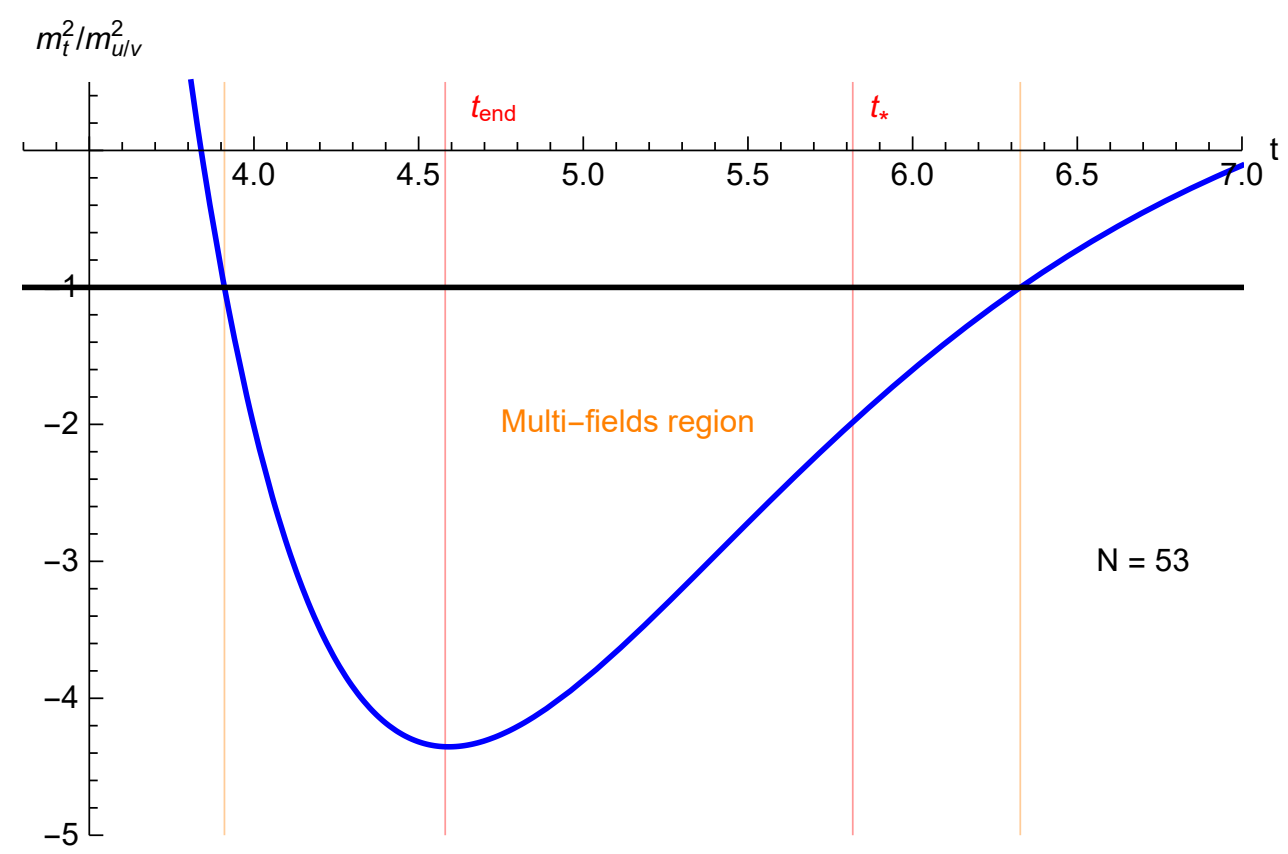

Figure 5: The mass ratio between the inflaton $t$ and other two Kähler modulus for $n_{s}=0.9605, N_{*}=53$. The multi-field region is among the two orange lines which covers the whole inflation period.

The existence of a multi-field region brings the question of possible large non-gaussianities. Here, in both cases, we assume that the moduli ratios are stabilised at the minimum during the whole period of inflation. There could be a deviation from the minimum at the initial time $t_{*}$. In the case of $N_{*}=60$ above, since inflaton is the lightest scalar at $t^{*}$, one can set the initial condition of deviation to be small. The mass of the ratios are much below the Hubble expansion rate $H$, so that the Hubble damping will take them back to their minimum without oscillations. The fraction of the kinetic energy of the moduli ratios thus keeps decreasing. From [25], this leads to negligible multi-field effects in the non-gaussianity signals. One can treat them as effective single field inflation. In the case of $N_{*}=53$, the trajectory we choose is still effectively single field inflation. However the initial condition is more fine-tuned since the inflaton at $t^{*}$ is already heavier than the ratio moduli.

\section{Discussion and Conclusions}

In the present work, slow roll inflation has been studied in the framework of type II-B/F theory effective supergravity using the six-dimensional compactification volume modulus as the inflaton. The analysis is performed in the context of a recently proposed framework of moduli stabilisation [8], in which a geometric configuration of three intersecting stacks of D7 branes is considered. In this set up, perturbative string loop contributions induce terms in the Kähler potential which depend logarithmically on the volume moduli associated with the directions transverse to the corresponding D7 branes. These contributions, together with the -already well known- $\alpha^{\prime}$ corrections, break the no-scale invariance of the Kähler potential. Also, the aforementioned D7 branes with magnetic fluxes induce positive definite D-terms in the scalar potential. These two elements, the logarithmic loop-corrections and the positive D-terms, are sufficient to stabilise the Kähler moduli. At the same time, they ensure a de Sitter minimum with a tiny positive cosmological constant. It is worth emphasising that this geometric stabilisation 
method, does not require inclusion of non-perturbative effects in the superpotential. The dS vacua generated with the use of the above ingredients only, are restricted in a small region of the available parameter space. Then, regarding the implementation of a successful cosmological inflationary scenario, it can be easily realised that this is not possible because the predicted number of e-foldings $N_{*}$ are found to be around an order of magnitude smaller compared to the anticipated value $N_{*} \approx 60$.

As a result, it seems that within our perturbative large volume approximation, one needs a new source of uplifting the vacuum energy. In this work we considered the effect of a new FI contribution to the scalar potential written recently in global and local supersymmetry which is manifestly gauge invariant at the Lagrangian level and, thus, avoiding the requirement of standard supergravity to be associated to a $U(1)$ R-symmetry [14, 15, 16]. This opens also the possibility of being generated as an effective term in string compactifications, although this is not clear at present. Alternatively, we considered a class of uplifting potentials arising from a nilpotent superfield and depending on some rational modular weights. A particular choice reproduces the effect of the new FI term. Although realistic inflation can be obtained in several cases, here we presented a detailed analysis for the simplest case that describes also the new FI term. When the latter is included in the scalar potential, new metastable de Sitter vacua can be generated which are suitable for slow-roll cosmological inflation. More precisely, the effective potential derived in this geometric set-up depends on three unspecified parameters multiplying an equal number of simple distinct elements: the first two are the strentghs of the F-term (which includes the $\alpha^{\prime}$ and the logarithmic string loop corrections) and the D-term, and the third parameter is associated with the new FI-term. Moreover, F- and D-terms depend on the three Kähler moduli related to the three intersecting D7 branes.

The inflaton field is identified with the logarithm of the internal $6 d$-volume, and it is proportional to the sum of the three canonically normalised Kähler moduli. This choice is suggestive for a new basis, in which the remaining two degrees of freedom (combinations of the Kähler moduli) are taken to be 'orthogonal' to the total volume. F- and D-terms are instrumental in defining the mass spectrum of the three moduli fields via the minimisation procedure, while the new FI-term ensures a sufficiently positive vacuum energy to accommodate slow-roll inflation. After implementing the constraints imposed by the minimisation conditions, the scalar potential obtains a simple form. In the large volume limit all slow roll conditions are satisfied by fitting the three available parameters. The model predicts a relatively small value for the tensor-to-scalar ratio parameter $(r \sim 0.0002)$ which is far beyond the required values for observation. Although a definite clue that inflation has a string theory origin is still lacking, the successful implementation of the slow-roll inflation in the present model provides a very compelling argument for the relevance of string theory to cosmology.

\section{Acknowledgements}

This work was supported in part by the Swiss National Science Foundation, in part by Labex "Institut Lagrange de Paris" and in part by a CNRS PICS grant. G.K.L. would like to thank the LPTHE in Paris and the ITP in Bern for their kind hospitality while Y.C. would like to thank ITP, where part of the work was completed. Y.C. also thanks Taro Mori for help and useful discussions. 


\section{References}

[1] R. Kallosh, Lect. Notes Phys. 738 (2008) 119 [hep-th/0702059].

S.-H. Henry Tye, Lect. Notes Phys. 737 (2008) 949 [hep-th/0610221].

D. Baumann and L. McAllister, "Inflation and String Theory," arXiv:1404.2601.

M. Cicoli and F. Quevedo, Class. Quant. Grav. 28 (2011) 204001 arXiv:1108.2659].

[2] S. Kachru, R. Kallosh, A. D. Linde and S. P. Trivedi, Phys. Rev. D 68 (2003) 046005 hep-th/0301240].

[3] V. Balasubramanian, P. Berglund, J. P. Conlon and F. Quevedo, JHEP 0503 (2005) 007 hep-th/0502058.

[4] U. H. Danielsson and T. Van Riet, Int. J. Mod. Phys. D 27 (2018) no.12, 1830007 arXiv:1804.01120 [hep-th]].

G. Obied, H. Ooguri, L. Spodyneiko and C. Vafa, arXiv:1806.08362 [hep-th].

[5] P. Agrawal, G. Obied, P. J. Steinhardt and C. Vafa, Phys. Lett. B 784 (2018) 271 arXiv:1806.09718 [hep-th]].

Y. Akrami, R. Kallosh, A. Linde and V. Vardanyan, Fortsch. Phys. 20181800075 arXiv:1808.09440 [hep-th]].

M. Cicoli, S. De Alwis, A. Maharana, F. Muia and F. Quevedo, arXiv:1808.08967 [hep-th]. J. J. Heckman, C. Lawrie, L. Lin and G. Zoccarato, arXiv:1811.01959 [hep-th].

J. J. Heckman, C. Lawrie, L. Lin, J. Sakstein and G. Zoccarato, arXiv:1901.10489 [hep-th].

[6] J. P. Conlon and F. Quevedo, JHEP 0601, 146 (2006) hep-th/0509012.

[7] M. Cicoli, C. P. Burgess and F. Quevedo, JCAP 0903, 013 (2009) arXiv:0808.0691 [hepth]].

[8] I. Antoniadis, Y. Chen and G. K. Leontaris, Eur. Phys. J. C 78 (2018) no.9, 766 arXiv:1803.08941 [hep-th]].

[9] I. Antoniadis and C. Bachas, Phys. Lett. B 450 (1999) 83 hep-th/9812093.

[10] A. Saltman and E. Silverstein, JHEP 0411 (2004) 066 hep-th/0402135.

[11] C. P. Burgess, R. Kallosh and F. Quevedo, JHEP 0310 (2003) 056 [hep-th/0309187].

[12] K. Dasgupta, C. Herdeiro, S. Hirano and R. Kallosh, Phys. Rev. D 65 (2002) 126002 hep-th/0203019].

[13] P. Fayet and J. Iliopoulos, Phys. Lett. 51B (1974) 461.

[14] N. Cribiori, F. Farakos, M. Tournoy and A. van Proeyen, JHEP 1804, 032 (2018) arXiv:1712.08601 [hep-th]].

[15] I. Antoniadis, A. Chatrabhuti, H. Isono and R. Knoops, "Fayet-Iliopoulos terms in supergravity and D-term inflation," Eur. Phys. J. C 78 (2018) no.5, 366 arXiv:1803.03817 [hep-th]].

[16] I. Antoniadis, A. Chatrabhuti, H. Isono and R. Knoops, arXiv:1805.00852 [hep-th].

[17] K. Becker, M. Becker, M. Haack and J. Louis, JHEP 0206 (2002) 060 hep-th/0204254. 
[18] D. Z. Freedman and A. Van Proeyen, "Supergravity," Cambridge, UK: Cambridge Uni. Pr. (2012) 607p.

[19] F. Farakos, A. Kehagias and A. Riotto, "Liberated $\mathcal{N}=1$ supergravity," JHEP 1806 (2018) 011 arXiv:1805.01877[hep-th]].

[20] Y. Aldabergenov, S. V. Ketov and R. Knoops, Phys. Lett. B 785 (2018) 284 arXiv:1806.04290 [hep-th]].

[21] E. Dudas, C. Papineau and S. Pokorski, JHEP 0702, 028 (2007) hep-th/0610297].

[22] H. Abe, T. Higaki, T. Kobayashi and Y. Omura, Phys. Rev. D 75, 025019 (2007) hepth/0611024.

[23] L. E. Ibanez and A. M. Uranga, "String theory and particle physics: An introduction to string phenomenology,"

[24] Y. Akrami et al. [Planck Collaboration], arXiv:1807.06211 [astro-ph.CO].

[25] C. T. Byrnes, K. Y. Choi and L. M. H. Hall, JCAP 0810, 008 (2008) arXiv:0807.1101 [astro-ph]]. 